\title{
HIGH DOSE FAST NEUTRON DOSIMETRY USING PADC PLASTIC NUCLEAR TRACK DETECTORS AND GREY LEVEL ANALYSIS
}

\author{
A. Stabilini ${ }^{1,2}$, K. Meier ${ }^{1}$ and E. G. Yukihara ${ }^{1,3, *}$ \\ ${ }^{1}$ Department of Radiation Safety and Security, Paul Scherrer Institute, 5232 PSI Villigen, Switzerland \\ ${ }^{2}$ Politecnico di Milano, Dipartimento di Energia, via la Masa 3420156 Milan, Italy \\ ${ }^{3}$ Physics Department, Oklahoma State University, Stillwater, OK 74078, USA \\ *Corresponding author: eduardo.yukihara@psi.ch
}

\begin{abstract}
The objective of this work is to demonstrate the possibility of performing fast neutron dosimetry up to $5 \mathrm{~Sv}$ using optical absorbance of polyallyl diglycol carbonate (PADC) detectors, obtained through grey level analysis of PADC images acquired with a commercial track-counting dosimetry system, and estimate the uncertainties involved. PADCs were irradiated with doses from $100 \mathrm{mSv}$ to $5 \mathrm{~Sv}\left({ }^{252} \mathrm{Cf}\right.$ source) and etched. PADC images were acquired using the TASLIMAGE ${ }^{\mathrm{TM}}$ Neutron Dosimetry System (Track Analysis Systems Ltd.) and analysed to obtain the grey levels and the optical absorbance. The absorbance from different detectors and batches was analysed to determine the uncertainties involved, from which the final uncertainty in the method, $\sim \mathbf{3 0} \%$ and dominated by the uncertainty in the calibration curve, was estimated. A dose estimation $<2 \mathrm{~Sv}$ can also be performed using a 'universal curve' by normalising the absorbance to that of a detector irradiated with $1 \mathrm{~Sv}$. The data presented here allows the extension of the dose range of track counting systems using no additional equipment, only the images already acquired by the systems.
\end{abstract}

\section{INTRODUCTION}

Polyallyl diglycol carbonate (PADC) detectors are widely used for neutron dosimetry using counting of tracks created by secondary charged particles, enlarged by a chemical etch. Analysis of the individual tracks provides information on the neutron fluence and the linear energy transfer of charged particles in the detector ${ }^{(1)}$.

For neutron doses above $\sim 100 \mathrm{mSv}$, however, track overlap prevents individual track counting. A correction was proposed to account for track overlap in PADC detectors ${ }^{(2,3)}$, but they were developed for Radon measurements ${ }^{(2)}$ or require Monte-Carlo simulations ${ }^{(3)}$, and have not been demonstrated for neutron dosimetry.

A simpler and more practical option for PADC high dose neutron dosimetry is to use optical absorption measurements ${ }^{(4,5)}$. Although absorbance measurements usually require a specialised spectrophotometer, they can also be done based on the PADC images obtained using commercial dosimetry systems. The radiationinduced tracks appear as dark spots in the images, reducing the image grey level ${ }^{(5)}$. Using the image from unirradiated and irradiated detectors, the absorbance can be calculated by the following equation:

$$
A=\log \left(\mathrm{I}_{0} / I\right)
$$

where, $I$ is the light intensity transmitted through the irradiated detector, and $I_{0}$, the light intensity transmitted through the unirradiated detector. The light intensity is given by the average grey level of the detector image. Although this procedure is relatively straightforward, its reproducibility and precision remains to be investigated.

The objective of this work is to demonstrate the possibility of performing fast neutron dosimetry up to $5 \mathrm{~Sv}$ using optical absorbance of PADC detectors, obtained through grey level analysis of PADC images acquired with a commercial track-counting dosimetry system, and estimate the uncertainties involved. PADCs were irradiated with doses from $100 \mathrm{mSv}$ to $5 \mathrm{~Sv}\left({ }^{252} \mathrm{Cf}\right.$ source $)$ and etched. PADC images were acquired using the TASLIMAGE ${ }^{\mathrm{TM}}$ Neutron Dosimetry System (Track Analysis Systems Ltd.) and analysed to obtain the grey levels and the optical absorbance. The absorbance from different detectors and batches was analysed to determine the uncertainties, from which the final uncertainty in the method was estimated. We should emphasise that the estimated uncertainties do not include those related to the neutron energy response, since this will influence the track size distribution and the change in grey level in the images ${ }^{(5)}$.

\section{METHODOLOGY}

PADC TASTRAK ${ }^{\mathrm{TM}}$ detectors manufactured by Track Analysis System Ltd. (TASL), of $20 \mathrm{~mm} \times 25 \mathrm{~mm} \times$ $1.5 \mathrm{~mm}$ dimension were used in this study. For irradiation the detectors were placed in custom-made 
HIGH DOSE FAST NEUTRON DOSIMETRY USING PADC

polyamide holders of $2 \mathrm{~mm}$ thickness on both sides of the detector, which works primarily as a fast neutron converter ${ }^{(6)}$, but also has a response to thermal neutrons due to the ${ }^{14} \mathrm{~N}(\mathrm{n}, \mathrm{p})$ neutron capture reaction.

The neutron irradiations were performed at PSI's accredited secondary standards calibration laboratory using a ${ }^{252} \mathrm{Cf}$ source ${ }^{(7)}$. Two irradiation series were performed: in the first series, detectors of the same batch were exposed to various doses on a panoramic irradiator calibrated in terms of $H_{p}(10)$. In the second series, carried out to estimate batch-tobatch variations in the signal, detectors from different batches were irradiated on the same carousel. For every series, unirradiated detectors were analysed to estimate the zero dose grey level, and six detectors were used for each dose.

The PADCs were etched using the standard conditions employed in routine analysis at PSI, consisting in a solution $6.25 \mathrm{M}$ of $\mathrm{NaOH}$ at $85^{\circ} \mathrm{C}$ for $2 \mathrm{~h}$ and $50 \mathrm{~min}^{(8)}$. After etching the PADCs were neutralised for $30 \mathrm{~min}$ in a $2 \%$ solution of acetic acid $\left(\mathrm{C}_{2} \mathrm{H}_{4} \mathrm{O}_{2}\right)$ and distilled water at room temperature, and subsequently washed for $30 \mathrm{~min}$ in pure water at room temperature. For the PADC readout we used the TASLIMAGE ${ }^{\mathrm{TM}}$ Neutron Dosimetry System (Track Analysis Systems Ltd.), which consists of a CCD camera mounted on an optical microscope, beneath which a motorised stage allows the automatic detector scanning. For each detector 315 images were acquired.

The grey level from each pixel, which ranges from zero (black) to one (white), was averaged over the entire image, and over the set of images of each detector to calculate the absorbance $(A)$ as in Eq. (1). Calibration curves were obtained by plotting the grey level or the absorbance versus irradiation dose. The absorbance versus dose was fitted with a saturating exponential function:

$$
A=a\left(1-e^{-b D}\right)
$$

where, $a$ and $b$ are the fitting parameters, and $D$ is the given dose.

The uncertainty in the measured dose was estimated taking into account the uncertainties in the detector grey level and in the absorbance calculation, and the uncertainty in the calibration curve. The final uncertainty can be written as follows:

$$
u_{\mathrm{D}}^{2}=u_{\mathrm{cal}}^{2}+u_{\text {meas }}^{2}
$$

where, $u_{\text {cal }}$ is the contribution due to uncertainties in the fitted calibration curve $\left(u_{a}^{2}\right.$ and $u_{b}^{2}$ and covariance $\operatorname{cov}(a, b)$ of the fitted parameters $a$ and $b)$ :

$$
\begin{aligned}
u_{\mathrm{cal}}^{2}= & \left(\frac{\partial D}{\partial a}\right)^{2} u_{a}^{2}+\left(\frac{\partial D}{\partial b}\right)^{2} u_{b}^{2} \\
& +\left(\frac{\partial D}{\partial a}\right)\left(\frac{\partial D}{\partial b}\right) \operatorname{cov}(a, b)
\end{aligned}
$$

and $u_{\text {meas }}$ is the contribution due to the uncertainty in the absorbance measurement:

$$
u_{\text {meas }}^{2}=(\partial D / \partial A)^{2} u_{A}^{2}
$$

For each detector the uncertainty in the absorbance can be estimated by error propagation, based on Eq. (1).

The uncertainties in the grey level have two major contributions: (a) variability in the grey level over the detector area (i.e. image to image), and (b) detector-to-detector variability within the same batch. If detectors from different batches are used, then batch to batch variability must be taken into account.

\section{RESULTS AND DISCUSSION}

Figure 1 shows examples of PADC grey level histograms. The average grey level decreases as the detector darkens due to increasing track density. The unirradiated detectors have a mean grey level of 0.58 (FWHM $\sim 0.026$ ), whereas the additional radiationinduced tracks introduce a peak centred at $\sim 0.28$ (FWHM 0.25).

Figure 2 shows the grey level versus dose for each batch. The standard deviation of the grey level within the same batch was $<0.6 \%$, but the difference between maximum and minimum value at $1 \mathrm{~Sv}$ was $6.2 \%$ for different batches. The zero dose grey level was very similar for all batches, with a difference between the maximum and the minimum of $0.9 \%$, which means that unirradiated detectors from a different batch can be used to calculate the absorbance.

The inset in Figure 2 shows the absorbance versus dose fitted with Eq. (2). The fitted curve describes well the dose for each group, allowing an absorbed dose estimation for detectors of the same batch exposed to unknown high doses.

The data in Figure 2 indicates that the batch-tobatch variations are large, preventing the use of a single calibration curve for different batches. An attempt was made to correlate the batch grey level sensitivity with other parameters (e.g. track density sensitivity at low doses), without success.

To investigate the contribution from different sources of uncertainty to the final absorbance uncertainty, Figure 3a shows the single-image grey level uncertainty of a single detector $\left(u_{\text {image }}\right)$, estimated as the standard deviation of the mean grey level of 315 images of a single detector. The figure also shows the 

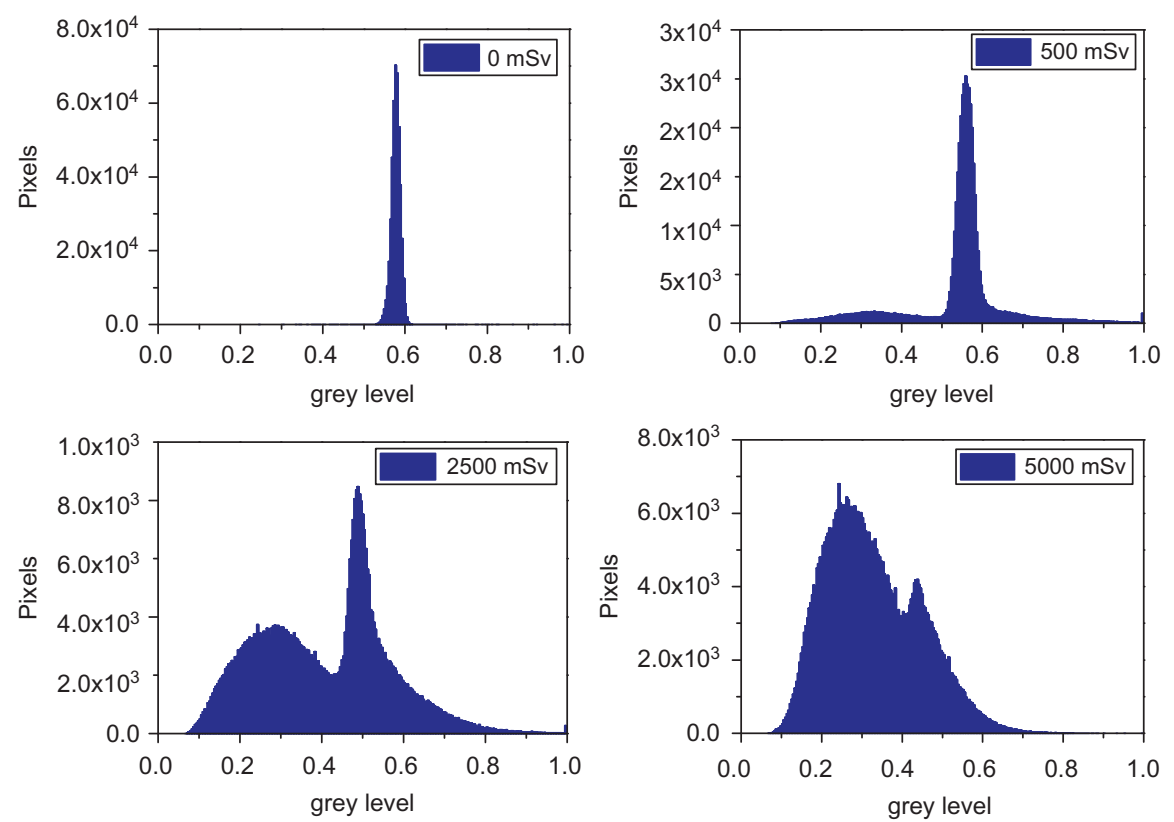

Figure 1. Grey level histograms for images of PADC detector irradiated with various doses.

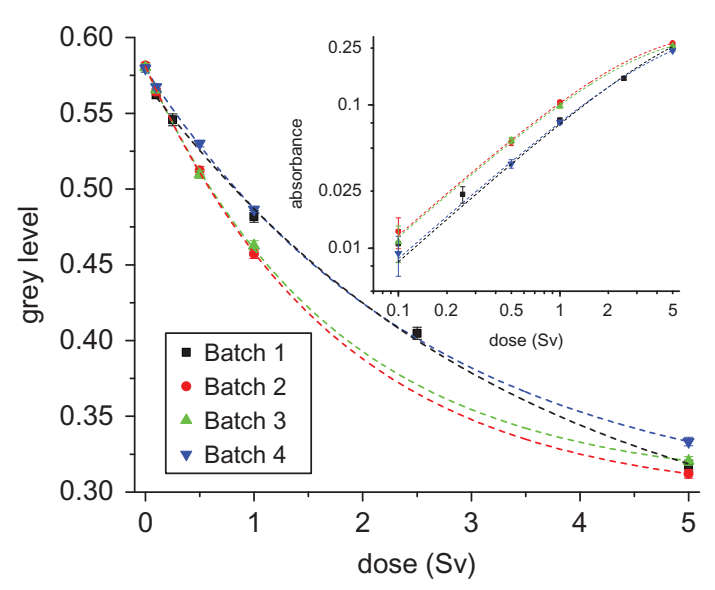

Figure 2. Calibration curves represented in terms of mean grey level or mean absorbance (inset) versus dose. The error bars are the standard deviation of detectors of the same batch.

uncertainty in the mean grey level of 315 images $\left(u_{\text {mean }}\right)$, determined as $u_{\text {mean }}=u_{\text {image }} / \sqrt{N_{\text {images }}}$, and, finally, the uncertainty due to detector-to-detector variability within the same batch $\left(u_{\mathrm{det}}\right)$, estimated by the standard deviation of the mean grey level for a group of six detectors.

Although the uncertainty based on a single image $\left(u_{\text {image }}\right)$ is higher than the detector-to-detector variability $\left(u_{\mathrm{det}}\right)$, the uncertainty in the mean grey level for each detector $\left(u_{\text {mean }}\right)$, calculated using the complete set of images (315), is negligible (one order of magnitude lower) than the detector-to-detector uncertainty $u_{\text {det. }}$. Therefore, it is recommended to use a large number of images to determine the grey level. This is normally not a problem, since the readout requires $<1 \mathrm{~min}$ per detector, including the acquisition of the 315 images.

Figure $3 \mathrm{a}$ also shows that the uncertainty in the grey level within different detectors of the same batch increases with dose. This was parameterised using a linear fit in the log-log scale (see fitted parameters in figure caption).

Using this data, it is possible to estimate the uncertainty in the absorbance calculation using Eq. (1) and standard error propagation. Figure $3 b$ shows the final uncertainty in the absorbance, estimated by the standard deviation of the absorbance for a group of six detectors, where $I_{0}$ was taken as the mean grey level of six unirradiated detectors of the same batch. The final uncertainty in the optical absorption as a function of dose is given by the dashed line and describes well the observed behaviour for the various batches.

Based on the above estimated uncertainties, Table 1 shows typical uncertainties at different stages of the calculation for various dose levels: $u_{\mathrm{det}}$ refers to the uncertainty in the grey level for one detector, considering the detector-to-detector variability, $u_{A}$ is the uncertainty based on the absorbance calculation 

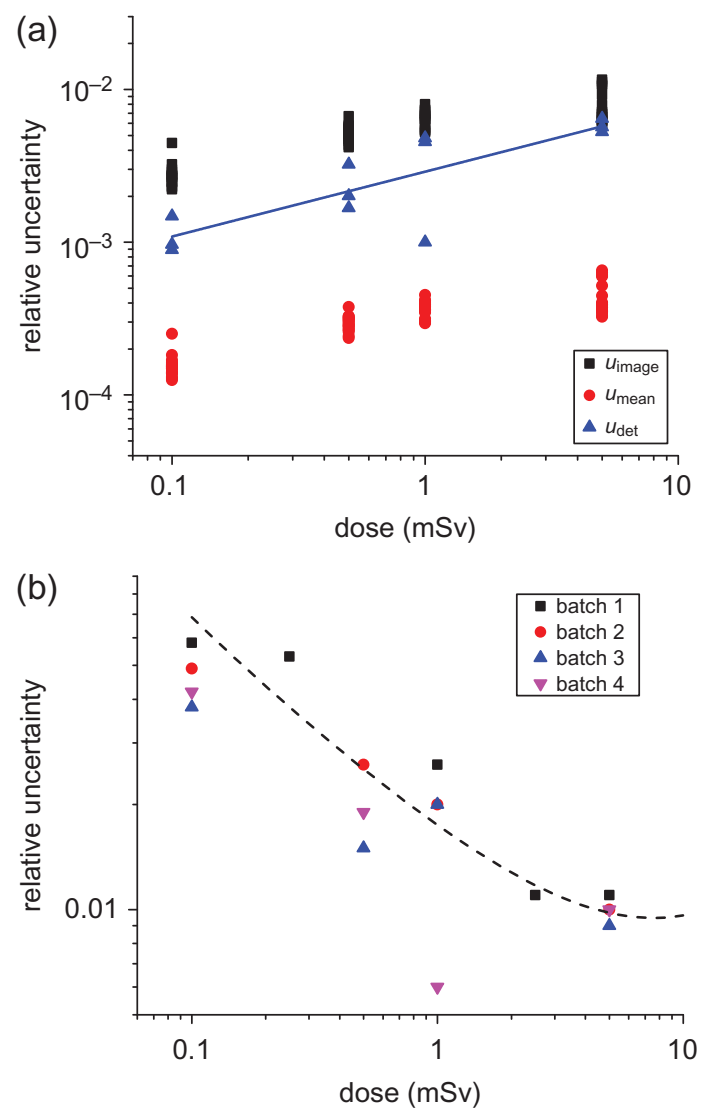

Figure 3. (a) Relative uncertainties in the image grey levels, in the detector grey level, and in the detector absorbance; the detector grey level data were fitted with the equation log $\left(u_{\mathrm{det}}\right)=0.425 \log (D-3.812)$; and (b) relative uncertainties in the absorbance for different batches.

Table 1. Relative uncertainties associated with the dosimetry using grey level analysis of a batch of PADC detectors.

\begin{tabular}{lllll}
\hline Source & $0.1 \mathrm{~Sv}$ & $0.5 \mathrm{~Sv}$ & $1 \mathrm{~Sv}$ & $5 \mathrm{~Sv}$ \\
\hline$u_{\text {det }}$ & $0.12 \%$ & $0.21 \%$ & $0.38 \%$ & $0.60 \%$ \\
$u_{\mathrm{A}}$ & $6.9 \%$ & $2.5 \%$ & $1.7 \%$ & $1.0 \%$ \\
$u_{\text {meas }}$ & $7.2 \%$ & $2.7 \%$ & $1.9 \%$ & $1.7 \%$ \\
$u_{\text {cal }}$ & $26 \%$ & $26 \%$ & $27 \%$ & $34 \%$ \\
$u_{\mathrm{D}}$ & $27 \%$ & $27 \%$ & $27 \%$ & $34 \%$ \\
\hline
\end{tabular}

(Figure 3b), considering that six detectors were used to determine $I_{0}$ and one detector was used to obtain $I, u_{\text {meas }}$ is the uncertainty associated with converting the absorbance to dose [Eq. (5)], and $u_{\text {cal }}$ is the uncertainty associated with the calibration curve [Eq. (4)]. The table shows that the main contribution to the final uncertainty on the dose $\left(u_{D}\right)$ comes from the calibration curve.

We tried to establish a universal calibration curve that could be used for all batches, by normalising the absorbance of each batch to the absorbance at $1 \mathrm{~Sv}$.

Figure 4 shows that, with this procedure, a single calibration curve can be defined, although the values at low doses $(100 \mathrm{mSv})$ are still very uncertain because of the small grey level, and the values at high doses $(5 \mathrm{~Sv})$ are uncertain due to different saturation level of the batches. This demonstrates that, at least for the $100 \mathrm{mSv}-2 \mathrm{~Sv}$ range, dosimetry can be performed using this calibration curve, as long as a normalisation value at $1 \mathrm{~Sv}$ is obtained. This eliminates the need to obtain an entire calibration curve for each dosimeter batch, but the uncertainties would be of course larger.

As a test, the procedure was applied to a set of detectors irradiated with neutrons at different known doses. Table 2 summarises the assessed dose values $(M)$ along with its uncertainty and the reference values $(D)$. The estimated doses are in good agreement with the reference ones for every detector tested. The maximum discrepancy was $28 \%$ for a $250 \mathrm{mSv}$ dose level, reducing to less than $10 \%$ for higher doses. At the $250 \mathrm{mSv}$ dose level, however, a second estimate can also be made still by track counting, although corrections for track overlap may be necessary. In the same table are also displayed the intermediate quantities computed in the analysis, i.e. the grey level $(I)$ and the absorbance $(A)$, whose uncertainty $\left(u_{a}\right)$ was calculated using Eq. (1) and error propagation.

\section{CONCLUSIONS}

This study demonstrates the possibility of performing neutron dosimetry in the $100 \mathrm{mSv}-5 \mathrm{~Sv}$ range

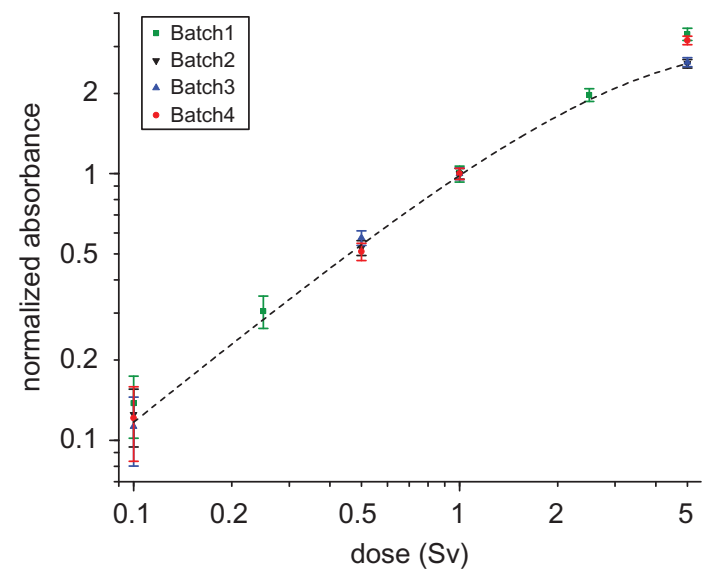

Figure 4. Calibration curve obtained by normalising all absorbance values by the value at $1 \mathrm{~Sv}$ for the respective batch. 


\section{A. STABILINI ET AL.}

Table 2. Sample detector of known delivered dose. The uncertainties were estimated as described in the previous Sections. The number in parentheses is the numerical value of the combined standard uncertainty $u_{c}$ referred to the corresponding last digits of the quoted result.

\begin{tabular}{lllcl}
\hline Detector & $D(\mathrm{~Sv})$ & $I$ & $A$ & $M(\mathrm{~Sv})$ \\
\hline 7045635 & 0.25 & $0.545(1)$ & $0.025(1)$ & $0.32(8)$ \\
7045648 & 1.0 & $0.481(2)$ & $0.079(2)$ & $1.08(30)$ \\
7045653 & 2.5 & $0.407(2)$ & $0.152(2)$ & $2.3(7)$ \\
7045660 & 5.0 & $0.321(2)$ & $0.256(3)$ & $5.0(17)$ \\
\hline
\end{tabular}

using the grey level analysis of PADC images acquired using a commercial track counting dosimetry system. The detector absorbance, calculated based on the mean grey level of a set of images for each detector, showed a linear-saturation behaviour with dose. The final uncertainties in the dose estimation are $\sim 30 \%$ and are dominated by uncertainties in the calibration curve parameters. A dose estimation $<2 \mathrm{~Sv}$ can also be performed using a 'universal curve' by normalising the absorbance to that of a detector irradiated with $1 \mathrm{~Sv}$. This simple method allows the extension of the dose range of track counting systems using no additional equipment, just using images already acquired by the systems. More sophisticated analysis could also be developed to improve the dosimetry in the low dose range (e.g. by restricting the analysis to the grey level range $<0.4$; see Figure 1), but this is not needed due to the possibility of using track counting in this dose range. The study was carried out for a ${ }^{252} \mathrm{Cf}$ neutron source and the influence of the neutron spectrum was not investigated.

\section{ACKNOWLEDGMENTS}

The authors thank Helga Schröter for the chemical etching of the detectors, and the Swiss Nuclear Safety Inspectorate (contract no. H-101 196) for the support.

\section{REFERENCES}

1. Cassou, R. M. and Benton, E. V. Properties and applications of CR-39 polymeric nuclear track detector. Nucl. Track Detection 2(3), 173-179 (1978).

2. Franci, D. and Aureli, T. A method to account for track overlap in CR-39 detectors. Radiat. Prot. Dosimetry 158 (1), 107-110 (2014).

3. Zylstra, A. B. et al. A new model to account for track overlap in CR-39 data. Nucl. Instrum. Methods Phys. Res. Sec. A 681, 84-90 (2012).

4. Bordy, J. M., Medioni, R. and Portal, G. Spectrophotometric measurements of track density in CR39 for high neutron dose determinations. Nucl. Tracks Radiat. Meas. 19(1-4), 241-244 (1991).

5. d'Errico, F., Weiss, M., Luszik-Bhadra, M., Matzke, M., Bernardi, L. and Cecchi, A. A CR-39 track image analyser for neutron spectrometry. Radiat. Meas. 28(1-6), 823-830 (1997).

6. Boschung, M., Fiechtner, A., Mayer, S. and Wernli, C. Field calibration and comparison of personal neutron dosemeter designs based on CR-39 for the use around high-energy accelerators. Radiat. Meas. 43(2-6), 1081-1084 (2008).

7. Hoedlmoser, H., Schuler, C., Butterweck, G. and Mayer, S. Characteristics of the neutron irradiation facilities of the PSI calibration laboratory. AIP Conf. Proc. 1412, 385-392 (2011).

8. Mayer, S., Assenmacher, F. and Boschung, M. Determination of the response function for two personal neutron dosemeter designs based on PADC. Radiat. Prot. Dosimetry 161(1-4), 82-85 (2014). 